Article

\title{
Primary Granulocyte Colony-Stimulating Factor
} Prophylaxis in Metastatic Pancreatic Cancer Patients Treated with FOLFIRINOX as the First-Line Treatment

Jae Hyup Jung ${ }^{1}$, Dong Woo Shin ${ }^{2}$, Jaihwan Kim ${ }^{1}$, Jong-Chan Lee ${ }^{1,3, *}$ and Jin-Hyeok Hwang ${ }^{1,3, *}$

1 Department of Internal Medicine, Seoul National University College of Medicine, Seoul National University Bundang Hospital, Seongnam 13620, Korea; 54279@snubh.org (J.H.J.); drjaihwan@snubh.org (J.K.)

2 Division of Gastroenterology, Department of Internal Medicine, Keimyung University School of Medicine, Keimyung University Dongsan Medical Center, Daegu 42601, Korea; delight0618@dsmc.or.kr

3 Division of Gastroenterology, Department of Internal Medicine, Seoul National University Bundang Hospital 82, Gumi-ro 173 Beon-gil, Bundang-gu, Seongnam-si, Gyeonggi-do, Seongnam 13620, Korea

* Correspondence: ljc0316@snubh.org (J.-C.L.); woltoong@snu.ac.kr (J.-H.H.); Tel.: +82-31-787-7043 or +82-10-6656-8588 (J.-C.L.); +82-31-787-7017 or +82-10-2613-9339 (J.-H.H.); Fax: +82-31-787-4051 (J.-C.L. \& J.-H.H.)

Received: 25 September 2020; Accepted: 24 October 2020; Published: 27 October 2020

Simple Summary: Because FOLFIRINOX shows high prevalence of hematologic toxicity, it is meaningful to investigate of usefulness of primary G-CSF prophylaxis in metastatic pancreatic cancer patients. In this retrospective study, a total of 165 patients were divided into G-CSF user group $(n=57)$ and non-user group $(n=105)$. Baseline characteristics were not significantly different between two groups, which included initial absolute neutrophil counts and metastatic burden. Primary G-CSF prophylaxis reduced the risk of neutropenia (55.6\% to $31.6 \%, p=0.003)$ and febrile neutropenia (18.5\% to $1.8 \%, p=0.002$ ) and improved OS (8.8 to 14.7 months; hazard ratio (HR): $1.766,95 \%$ CI: $1.257-2.481, p=0.001)$. When administering FOLFIRINOX for MPC, primary G-CSF prophylaxis could be rationalized to reduced AEs and improve survival, and more prospective studies are needed.

\begin{abstract}
Although FOLFIRINOX (5-fluorouracil, leucovorin, irinotecan, and oxaliplatin) has been proven efficacious in metastatic pancreatic cancer (MPC), physicians hesitate to administer it due to its hematologic toxicities. We investigated the usefulness of primary granulocyte colony-stimulating factor (G-CSF) prophylaxis. We reviewed electronic medical records of MPC patients with good performance status who were administered FOLFIRINOX as the first-line treatment from 2011 to 2017. The patients were divided into primary G-CSF prophylaxis users (group A) and non-users or therapeutic/secondary users (group B). Cumulative relative dose (cRDI), adverse effects (AEs), and overall survival (OS) were compared. A total of 165 patients (group A (57) vs. group B (108)) were investigated. Intergroup differences in baseline characteristics were not significant, although the cRDI and the number of treatment cycles were both higher in group A than in group B (cRDI: $80.6 \%$ vs. $73.9 \%, p=0.007 ; 9$ vs. 6 cycles, $p=0.004$ ). Primary G-CSF prophylaxis reduced the risk of neutropenia $(55.6 \%$ to $31.6 \%, p=0.003)$ and febrile neutropenia $(18.5 \%$ to $1.8 \%, p=0.002)$ and improved OS (8.8 to 14.7 months; hazard ratio [HR]: $1.766,95 \%$ CI: $1.257-2.481, p=0.001$ ). When administering FOLFIRINOX for MPC, primary G-CSF prophylaxis could be rationalized to reduced AEs and improve survival; more prospective studies are needed.
\end{abstract}

Keywords: pancreatic cancer; FOLFIRINOX; G-CSF prophylaxis 


\section{Introduction}

Pancreatic cancer (PC) was the fourth leading cause of death from cancer in both men and women in the United States in 2019 [1]. Although combination chemotherapy regimens such as FOLFIRINOX (5-fluorouracil, leucovorin, irinotecan, and oxaliplatin) have considerably contributed to improvements in the overall survival (OS) of patients with metastatic PC (MPC) since 2011, the overall five-year survival rate is $9 \%$, still indicating an unfavorable prognosis [2-4].

Since 2011, FOLFIRINOX is recommended as the first-line chemotherapy in MPC patients with good performance status [3,5]. Moreover, in 2019, modified FOLFIRINOX became a new a milestone in the adjuvant setting of resectable PC; consequently, the regimen is becoming more widely used [6]. However, FOLFIRINOX was demonstrated to show considerable hematologic and nonhematologic side effects, even with a dose-modified regimen. Therefore, modification of the FOLFIRINOX regimen while both maintaining efficacy and reducing toxicity remains an important issue [3,7-13].

In many retrospective studies, only $30-60 \%$ of PC patients who received FOLFIRINOX were administered primary granulocyte colony-stimulating factor (G-CSF) prophylaxis [14-16]. According to the National Comprehensive Cancer Network (NCCN) guidelines (version 2.2020) for the management of neutropenia, the risk group for febrile neutropenia among those treated with FOLFIRINOX for PC, changed from the intermediate-risk group to the high-risk group. However, routine primary G-CSF prophylaxis is still not recommended and is considered only in the high-risk population. Moreover, in some meta-analyses, primary G-CSF prophylaxis for solid tumors was associated with favorable OS [17-21].

Therefore, in the present study, we investigated the usefulness of primary G-CSF prophylaxis in reducing hematologic adverse effects (AEs) and improving survival in MPC patients with good performance status who were treated with FOLFIRINOX.

\section{Method}

\subsection{Study Population}

We collected the data of all patients with histologically diagnosed pancreatic ductal adenocarcinoma from October 2011 to December 2017 at a single tertiary teaching hospital (Seoul National University Bundang Hospital, Seoungnam-si, Korea). Among the patients whose data were collected, MPC patients treated with FOLFIRINOX as the first-line treatment were retrospectively evaluated in the present study. Patients with a poor performance status (Eastern Cooperative Oncology Group (ECOG) performance status score of $>2$ ) were excluded.

\subsection{Study Design}

The patients were divided into two groups according to whether primary G-CSF prophylaxis was administered or not, namely, primary G-CSF prophylaxis users (group A) and non-users (group B). Patients for whom only secondary G-CSF prophylaxis and therapeutic G-CSF were used were included in the non-user group (group B). To investigate the safety and efficacy of FOLFIRINOX administered as the first-line therapy, the cumulative relative dose intensity (cRDI), AEs, and OS were compared by reviewing electronic medical records. The data cut-off date was 30 June 2020. cRDI was measured by considering dose reduction and chemotherapy interval modification between the starting day of chemotherapy and the day of the first radiological evaluation [22]. The occurrence of grade 3 or 4 AEs within three months after initiating chemotherapy was compared based on Common Terminology Criteria for Adverse Events v5.0. OS was defined as the time from the histological diagnosis of MPC to death or the last follow-up. Survival data were evaluated until 30 June 2020.

\subsection{G-CSF Agents}

The G-CSF agent was used as filgrastim (subcutaneous, $300 \mathrm{mcg} /$ day, three to five days) or pegfilgrasim (a PEGylated form of the filgrastim, subcutaneous, $6 \mathrm{mg}$ per three weeks). The choice 
of two drugs was made according to the preference of the clinician, the requests of the patient, and the price.

\subsection{Statistical Analysis}

To compare the patients' baseline characteristics, T-, chi-square, and Fisher's exact tests were used. OS was evaluated using the Kaplan-Meier method and difference in survival was analyzed using the log-rank test. The Cox proportional hazard model was used to analyze survival and other factors. All tests were double-sided with a $p$-value of less than 0.05 for statistical significance. All analyses were performed using SPSS software version 22 (IBM corporation, New York, NY, USA).

\subsection{Ethics Statement}

The ethical approval for this study was obtained by the institutional review board of the Seoul National University Bundang Hospital, Seongnam, Korea, on 15 May 2020 (Approval Number B-2005/615-103).

\section{Results}

\subsection{Characteristics of the Patients}

A total of 165 patients (57 in group A vs. 108 in group B) met the eligibility criteria. Among them, 160 died during the follow-up period and three patients (one in group A vs. two in group B) remained alive until June 2020; the survival status of the remaining two patients (two in group A) was unknown. In group A, five patients were administered pegfilgrastim, while 52 were administered filgrastim.

The median age of the patients in both groups was 61.4 years; furthermore, $66.7 \%$ and $63.0 \%$ of the patients in groups A and B, respectively, were male. Intergroup differences (group A vs. group B) in pretreatment body mass index (BMI) (23.3 vs. $\left.22.7 \mathrm{~kg} / \mathrm{m}^{2}, p=0.147\right)$, serum albumin ( 4.1 vs. $3.9 \mathrm{~g} / \mathrm{dL}, p=0.544)$, and carbohydrate antigen (CA) $19-9$ level ( $660.0 \mathrm{vs.} 380.0 \mathrm{U} / \mathrm{mL}, p=0.138$ ) were not significant. The most common primary tumor site was the tail $(52.6 \%)$ in group A and the head and neck $(44.4 \%)$ in group B. Group A received a more intense FOLFIRINOX regimen ( $80.6 \%$ vs. $73.9 \%$ of cRDI, $p=0.007$ ) and for a longer duration compared to those in group B (9.0 vs. 6.0 cycles, $p=0.004)$. Initial absolute neutrophil count (ANC) level was not different between the two groups (Table 1).

Table 1. Baseline characteristics.

\begin{tabular}{|c|c|c|c|c|}
\hline Variables & 1st G-CSF User $(n=57)$ & 1st G-CSF Non-User $(n=108)$ & All Patients $(n=165)$ & $p$ Value \\
\hline Age, median (range, yr) & $61.1(41-80)$ & $61.5(29-79)$ & $61.4(29-80)$ & 0.856 \\
\hline Sex, no. $(\%)$ & & & & 0.637 \\
\hline Female & 19 (33.3) & $40(37.0)$ & $59(35.8)$ & \\
\hline Male & $38(66.7)$ & $68(63.0)$ & $106(64.2)$ & \\
\hline BMI, median (range, $\mathrm{kg} / \mathrm{m}^{2}$ ) & $23.3(15.4-29.1)$ & $22.7(15.0-29.3)$ & $23.0(15.0-29.3)$ & 0.147 \\
\hline ECOG performance status & & & & 0.095 \\
\hline $0-1$ & $51(89.5)$ & $104(96.3)$ & $155(93.9)$ & \\
\hline 2 & $6(10.5)$ & $4(3.7)$ & $10(6.1)$ & \\
\hline Primary site, no. (\%) & & & & 0.098 \\
\hline Head and neck & $20(35.1)$ & $48(44.4)$ & $68(41.2)$ & \\
\hline Body & $5(8.8)$ & $19(17.6)$ & $24(14.5)$ & \\
\hline Tail & $30(52.6)$ & $39(36.1)$ & $69(41.8)$ & \\
\hline Multicentric & $1(1.8)$ & $2(1.9)$ & $3(1.8)$ & \\
\hline Site of metastasis, no. $(\%)^{*}$ & & & & 0.874 \\
\hline Liver & $38(66.7)$ & $67(62.0)$ & $105(63.6)$ & \\
\hline Peritoneal seeding & $13(22.8)$ & $26(24.1)$ & 39 (23.6) & \\
\hline Distant lymph node & $17(29.8)$ & $21(19.4)$ & $38(23.0)$ & \\
\hline Lung & $12(21.1)$ & $20(18.5)$ & 32 (19.4) & \\
\hline Others & $13(22.8)$ & $21(19.4)$ & 34 (20.6) & \\
\hline
\end{tabular}


Table 1. Cont

\begin{tabular}{ccccc}
\hline Variables & 1st G-CSF User $(n=57)$ & 1st G-CSF Non-User $(n=108)$ & All Patients $(n=165)$ & $p$ Value \\
\hline Metastatic organ, no. (\%) & & & & 0.391 \\
2 & $32(56.1)$ & $72(66.7)$ & $107(64.8)$ & $37(22.4)$ \\
$\geq 3$ & $15(26.3)$ & $23(21.3)$ & $21(13.3)$ \\
\hline Baseline laboratory values & $10(17.5)$ & $13(12.0)$ & & \\
ANC, median (range, g/dL) & $4624(840-11,297)$ & $4723(1,331-12,178)$ & $4712(840-12,178)$ & 0.642 \\
Albumin, median (range, g/dL) & $4.1(2.5-4.7)$ & $3.9(2.4-5.0)$ & $3.9(2.4-5.0)$ & 0.544 \\
CA19-9, median (range, U/mL) & $660.0(5.0-20,000)$ & $380.0(5-20,000)$ & $464.6(5-20,000)$ & 0.138 \\
\hline cRDI, median (range) & $80.6(43.3-102.2)$ & $73.9(28.3-109.0)$ & $76.3(28.3-109.0)$ & 0.007 \\
Cycles, median (range) & $9.0(1-75)$ & $6.0(1-31)$ & $7.0(1-75)$ & 0.004 \\
\hline Data are presented as median (range) or number of patients/total number $(n \%)$, unless otherwise stated. * Site of \\
metastasis includes multiorgan involvement. G-CSF, granulocyte colony-stimulating factor; BMI, body mass index; \\
ECOG, Eastern Cooperative Oncology Group; ANC, absolute neutrophil count; CA 19-9, carbohydrate antigen; \\
cRDI, cumulative relative dose intensity; cRDI = (actual cumulative dose/standard cumulative dose) $\times 100$.
\end{tabular}

\section{2. $A E s$}

Neutropenia and febrile neutropenia were more frequently observed in group B $(31.6 \% \mathrm{vs}$. $55.6 \%, p=0.003 ; 12.7 \%$ vs. $18.5 \%, p=0.002$, respectively), while the incidence of anemia and thrombocytopenia did not differ between the groups. In terms of nonhematologic toxicities, there were no significant intergroup differences in the incidences of fatigue, vomiting, diarrhea, and peripheral sensory neuropathy (Table 2$)$. All patients who manifested neutropenic events $(n=60)$ received therapeutic G-CSF administration followed by secondary G-CSF prophylaxis.

Table 2. Serious adverse effect within three months.

\begin{tabular}{ccccc}
\hline Grade 3-4 Toxicity & $\begin{array}{c}\text { 1st G-CSF User } \\
(\boldsymbol{n}=\mathbf{5 7})\end{array}$ & $\begin{array}{c}\text { 1st G-CSF } \\
\text { Non-User }(\boldsymbol{n = 1 0 8 )})\end{array}$ & $\begin{array}{c}\text { All Patients } \\
(\boldsymbol{n}=\mathbf{1 6 5})\end{array}$ & $\boldsymbol{p}$ Value \\
\hline Hematologic & & & & \\
Neutropenia & $18 / 57(31.6)$ & $60 / 108(55.6)$ & $78 / 165(47.3)$ & 0.003 \\
Febrile neutropenia & $1 / 57(1.8)$ & $20 / 108(18.5)$ & $21 / 165(12.7)$ & 0.002 \\
Anemia & $3 / 57(5.3)$ & $17 / 108(15.7)$ & $20 / 165(12.1)$ & 0.050 \\
Thrombocytopenia & $2 / 57(3.5)$ & $9 / 108(8.3)$ & $11 / 165(6.7)$ & 0.237 \\
Nonhematologic & & & & \\
Fatigue & $1 / 56(1.8)$ & $5 / 105(4.8)$ & $6 / 161(3.7)$ & 0.342 \\
Vomiting & $3 / 56(5.4)$ & $13 / 105(12.4)$ & $16 / 161(9.9)$ & 0.156 \\
Diarrhea & $5 / 56(8.9)$ & $7 / 105(6.7)$ & $12 / 161(7.5)$ & 0.603 \\
Sensory & $0 / 56(0.0)$ & $2 / 105(1.9)$ & $2 / 161(1.2)$ & 0.299 \\
neuropathy & & & & \\
\hline
\end{tabular}

Data are presented as number of patients/total number (\%), unless otherwise stated. Grade 3-4 according to the Common Terminology Criteria for Adverse Events (CTCAE), version 4.03.

\subsection{Efficacy}

Analyses using unadjusted variables showed that the administration of primary G-CSF prophylaxis (14.7 vs. 8.8 months; hazard ratio (HR) 1.766; 95\% confidence interval (CI) 1.257-2.481) and normal albumin level (12.2 vs. 6.5 months; HR 1.864; 95\% CI 1.295-2.683) exerted significant beneficial effects on OS. However, there was no significant OS benefit afforded by the use of higher dose intensity ( $\geq 76.3$ of median cRDI) compared to that afforded by the lower dose ( $<76.3$ of median cRDI) (11.6 vs. 9.5 months; HR 1.183, 95\% CI 0.864-1.618). Old age, sex, and pretreatment CA 19-9 level were not identified as significant independent prognostic factors for OS. Analysis using adjusted variables showed that primary G-CSF prophylaxis (HR for death 1.799; 95\% CI 1.249-2.591) and normal albumin level (HR for death 1.718; 95\% CI 1.223-2.412) were independent favorable prognostic factors for OS (Table 3, Figure 1). 
Table 3. Unadjusted and adjusted relationships between administration of G-CSF with other variables and overall survival.

\begin{tabular}{|c|c|c|c|c|c|c|c|c|c|}
\hline \multirow{2}{*}{ Variables } & \multirow{2}{*}{$\begin{array}{l}\text { Number of } \\
\text { Patients (\%) }\end{array}$} & \multirow{2}{*}{$\begin{array}{c}\text { OS } \\
\text { (Median, Months) }\end{array}$} & \multirow{2}{*}{$\begin{array}{c}\text { 95\%CI } \\
\text { (Months) }\end{array}$} & \multicolumn{3}{|c|}{ Unadjusted } & \multicolumn{3}{|c|}{ Adjusted } \\
\hline & & & & HR & $95 \% \mathrm{CI}$ & $p$ Value & HR & $95 \% \mathrm{CI}$ & $p$ Value \\
\hline $\begin{array}{l}\text { Overall patients } \\
\text { Age }\end{array}$ & \multicolumn{8}{|c|}{ Age } & \\
\hline$<65 \mathrm{yr}$ & $102(62)$ & 11.4 & $9.3-13.5$ & & & & & & \\
\hline $\begin{array}{l}\geq 65 \mathrm{yr} \\
\text { Sex }\end{array}$ & $63(38)$ & 9.2 & $6.1-12.3$ & 1.154 & $0.837-1.591$ & 0.381 & & & \\
\hline Female & $59(36)$ & 11.6 & $7.8-15.3$ & & & & & & \\
\hline Male & $106(64)$ & 9.5 & $6.6-12.3$ & 1.297 & $0.936-1.797$ & 0.118 & & & \\
\hline \multicolumn{10}{|l|}{ Albumin } \\
\hline$\geq 3.5 \mathrm{~g} / \mathrm{dL}$ & $124(75)$ & 12.2 & $10.3-14.0$ & & & & & & \\
\hline $\begin{array}{l}<3.5 \mathrm{~g} / \mathrm{dL} \\
\text { CA } 19-9\end{array}$ & $41(25)$ & 6.5 & $4.3-8.7$ & 1.864 & $1.295-2.683$ & 0.001 & 1.718 & $1.223-2.412$ & 0.002 \\
\hline$<464.6 \mathrm{U} / \mathrm{mL}$ & $78(50)$ & 13.0 & $10.9-15.2$ & & & & & & \\
\hline $\begin{array}{l}\geq 464.6 \mathrm{U} / \mathrm{mL} \\
\text { Primary }\end{array}$ & $78(50)$ & 8.9 & $8.2-9.6$ & 1.166 & $0.845-1.607$ & 0.350 & & & \\
\hline \multicolumn{10}{|l|}{ G-CSF prophylaxis } \\
\hline Yes & $57(35)$ & 14.7 & $12.0-17.4$ & & & & & & \\
\hline No & $108(65)$ & 8.8 & 7.9-9.7 & 1.766 & $1.257-2.481$ & 0.001 & 1.799 & $1.249-2.591$ & 0.002 \\
\hline \multicolumn{10}{|l|}{ Dose intensity (cRDI) } \\
\hline$\geq 76.3$ & $83(50)$ & 11.6 & $8.1-15.2$ & & & & & & \\
\hline$<76.3$ & $82(50)$ & 9.5 & $7.1-11.8$ & 1.183 & $0.864-1.618$ & 0.295 & & & \\
\hline
\end{tabular}

Data are presented as median (range) or number of patients (\%), unless otherwise stated. OS denotes overall survival; CI, confidence interval; ECOG PS, Eastern Cooperative Oncology Group Performance Status; cRDI, cumulative relative dose intensity, cRDI = (actual cumulative dose $/$ standard cumulative dose $) \times 100$.

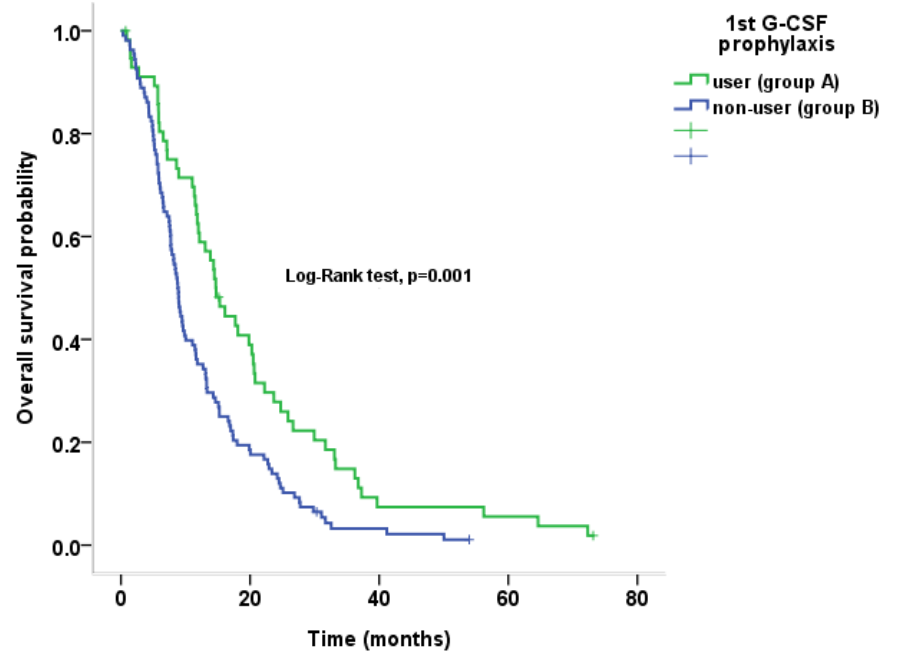

Figure 1. Overall survival in primary G-CSF users and non-users.

\subsection{Primary G-CSF Prophylaxis and Clinical Outcomes by Age Group}

Analysis of the treatment outcomes according to age groups (non-older patients [age $<65$ years] vs. older patients (age $\geq 65$ years)) showed that primary G-CSF prophylaxis exerted a significant beneficial effect on OS (14.5 vs. 8.7 months; HR 1.957, 95\% CI 1.258-3.042), while reducing the risk of neutropenia ( $38.9 \%$ vs. $60.6 \%, p=0.036)$ and febrile neutropenia $(0.0 \%$ vs. $16.7 \%, p=0.010)$ and increasing cRDI $(85.2 \%$ vs. $76.4 \%, p=0.007)$ in non-older patients (Table S1, Figure 2A). However, primary G-CSF prophylaxis did not have a significant effect on OS (14.7 vs. 8.8 months; HR 1.519; $95 \%$ CI $0.878-2.629)$, while reducing the risk of neutropenia $(19.0 \%$ vs. $47.6 \%, p=0.028)$ and not significantly reducing the risk of febrile neutropenia ( $4.8 \%$ vs. $21.4 \%, p=0.088$ ) with similar cRDI $(72.2 \%$ vs. $70.1 \%, p=0.334)$ in the older patient group. (Table S1, Figure $2 \mathrm{~B}$ ). 


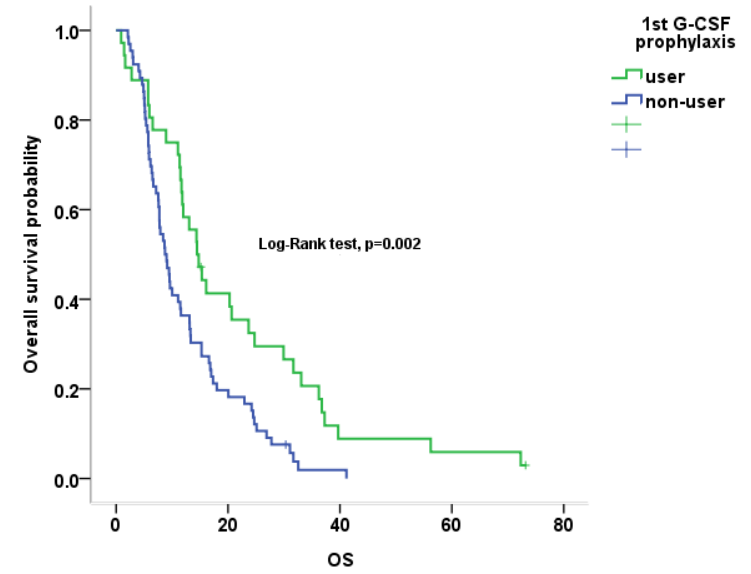

(A)

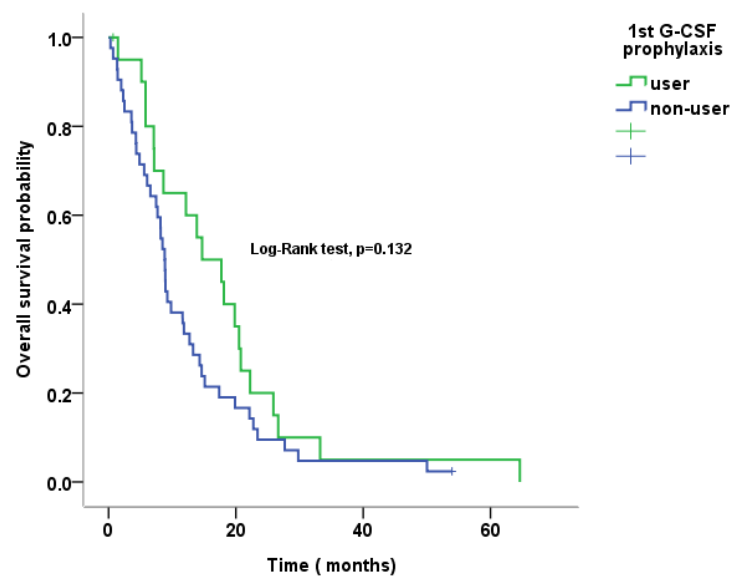

(B)

Figure 2. Overall survival in primary G-CSF users and non-users ((A) non-older patient subgroup; (B) older patient subgroup).

\section{Discussion}

In the present study, we attempted to investigate the routine use of primary G-CSF prophylaxis as a strategy for reduction of hematologic AEs and thus potentiation of the efficacy of FOLFIRINOX. We evaluated 165 patients with MPC, which was a relatively large number, and found that only $35.0 \%$ of the patients were administered primary G-CSF prophylaxis. Our results demonstrated that primary G-CSF prophylaxis reduced hematologic AEs (e.g., neutropenia and febrile neutropenia), and that an increase in dose intensity and treatment duration of FOLFIRINOX conferred a survival benefit in MPC patients. Similar to previous studies [17-21], our study demonstrated that routine primary G-CSF prophylaxis use is justified given its beneficial effects (considering the reduction in AEs in conjunction with the survival benefit).

Since it was recommended in 2011, FOLFIRINOX has considerably contributed to improving the survival of MPC patients [2-5,23]. Several retrospective and prospective studies showed that AEs due to FOLFIRINOX were more frequently observed compared to in the study by Conroy et al. [5,7,8,24,25]. Version 2.2020 of the NCCN guidelines emphasizes the risk of neutropenic fever associated with FOLFIRINOX administration in PC patients. Therefore, dose-modified regimens were studied in vulnerable patients; however, the efficacy of these regimens remains controversial [9-13].

According to the NCCN guidelines version 2.2019, FOLFIRINOX use is recommended for intermediate-risk patients for febrile neutropenia, and the administration of primary G-CSF prophylaxis may be recommended in patients aged 65 years or higher when FOLFIRINOX is prescribed at full-dose intensity. This recommendation was based on a randomized controlled trial conducted by Conroy et al., which showed that the rate of febrile neutropenia was only $5.4 \%$ in the FOLFIRINOX group (G-CSF was administered to $42.5 \%$ of the patients) [5]. However, several retrospective studies reported that febrile neutropenia was more frequently observed (14\% in KOREAN study [22] and $22.2 \%$ in a Japanese trial [24]) than reported in the randomized trial by Conroy et al. (5.4\%) in MPC patients who received FOLFIRINOX [5]. Although sufficient evidence was lacking, FOLFIRINOX was recommended for high-risk individuals for febrile neutropenia (febrile neutropenia risk of $>20 \%$ ) in the NCCN guidelines version 2.2020. Despite this revised risk stratification, routine primary G-CSF prophylaxis is not recommended for all populations and is only currently considered in the high-risk population. Our findings indicate that routine prophylactic G-CSF use might be more beneficial than expected.

In our study, we performed a subgroup analysis by age ( $<65$ years vs. $\geq 65$ years). Primary G-CSF prophylaxis reduced neutropenia in both subgroups ( $60.6 \%$ to $38.9 \%$ vs. $47.6 \%$ to $19.0 \%$, respectively). Contrary to expectations, primary G-CSF prophylaxis reduced febrile neutropenia in only the non-older group $(16.7 \%$ to $0.0 \%)$, although the older group showed a tendency toward febrile neutropenia 
reduction associated with primary G-CSF prophylaxis (21.4\% to $4.8 \%$ ). Furthermore, primary G-CSF prophylaxis conferred a significant survival benefit in only the non-older group, although the trend was similar in both groups, possibly because the number of older patients was too small to draw a conclusion. Another explanation could be that primary G-CSF prophylaxis had a favorable effect on survival as dose intensity was increased in the non-older group. In the older group, however, regardless of the administration of prophylactic G-CSF, the FOLFIRINOX dose intensity was similar. This perhaps reflects physicians' reluctance to initiate FOLFIRINOX at full-dose intensity because of concerns about AEs, despite the administration of primary G-CSF prophylaxis. Further studies are warranted to confirm these issues.

In the previous study, we found that greater dose reduction is needed in elderly patients with advanced pancreatic cancer when using FOLFIRINBOX [22], as reflected in Table S1. Since it was mentioned in previous studies that excessive dose reduction affects tumor response [22], it can be hypothesized that cRDI may also affect OS, which could be the subject of subsequent studies.

This study has several limitations. There is a possibility of selection bias because the patients were from a single tertiary teaching hospital. Furthermore, because there was no blinding, there is a possibility of performance bias by the physicians. Furthermore, this study did not consider the patients' socioeconomic status, which determines whether a patient receives primary G-CSF prophylaxis or not as the intervention is not reimbursed by national insurance in South Korea. Nevertheless, because the median patient age at diagnosis of PC has increased over the past 10 years ( 65 to 70 years), we believe that our results may be valuable when making a decision (primary G-CSF prophylaxis vs. a wait-and-see strategy) in older MPC patients treated with FOLFIRINOX.

\section{Conclusions}

In conclusion, primary G-CSF prophylaxis could improve survival when FOLFIRINOX is administered in MPC patients while reducing hematologic AEs, allowing for an increase in dose intensity and treatment duration of FOLFIRINOX. Thus, routine primary G-CSF prophylaxis could be justified in this patient population. More well-designed, prospective studies are necessary for large populations.

Supplementary Materials: The following are available online at http://www.mdpi.com/2072-6694/12/11/3137/s1, Table S1: Prophylactic G-CSF and clinical outcomes by age group.

Author Contributions: Conceptualization, J.H.J., J.-C.L. and J.-H.H.; methodology, J.H.J., J.K., J.-C.L. and J.-H.H.; software, J.H.J.; validation, J.H.J. and J.K.; formal analysis, J.H.J. and J.-C.L.; investigation, J.H.J.; resources, J.H.J. and D.W.S.; data curation, J.H.J. and D.W.S.; writing-original draft preparation, J.H.J.; writing-review and editing, J.-C.L. and J.-H.H.; supervision, J.-C.L. and J.-H.H.; project administration, J.-H.H. All authors have read and agreed to the published version of the manuscript.

Funding: This research received no external funding.

Conflicts of Interest: The authors declare no conflict of interest.

\section{References}

1. Siegel, R.L.; Miller, K.D.; Jemal, A. Cancer statistics, 2019. CA Cancer J. Clin. 2019, 69, 7-34. [CrossRef] [PubMed]

2. Rawla, P.; Sunkara, T.; Gaduputi, V. Epidemiology of pancreatic cancer: Global trends, etiology and risk factors. World J. Oncol. 2019, 10, 10-27. [CrossRef] [PubMed]

3. Neoptolemos, J.P.; Kleeff, J.; Michl, P.; Costello, E.; Greenhalf, W.; Palmer, H.D. Therapeutic developments in pancreatic cancer: Current and future perspectives. Nat. Rev. Gastroenterol. Hepatol. 2018, 15, 333-348. [CrossRef] [PubMed]

4. Perkhofer, L.; Ettrich, T.J.; Seufferlein, T. Pancreatic cancer: Progress in systemic therapy. Gastrointest. Tumors 2014, 1, 167-179. [CrossRef] [PubMed] 
5. Conroy, T.; Desseigne, F.; Ychou, M.; Bouché, O.; Guimbaud, R.; Bécouarn, Y.; Adenis, A.; Raoul, J.L.; Gourgou-Bourgade, S.; de la Fouchardière, C.; et al. Folfirinox versus gemcitabine for metastatic pancreatic cancer. N. Engl. J. Med. 2011, 364, 1817-1825. [CrossRef] [PubMed]

6. Conroy, T.; Hammel, P.; Hebbar, M.; Abdelghani, M.B.; Wei, A.C.; Raoul, J.L.; Choné, L.; Francois, E.; Artru, P.; Biagi, J.J.; et al. Folfirinox or gemcitabine as adjuvant therapy for pancreatic cancer. N. Engl. J. Med. 2018, 379, 2395-2406. [CrossRef] [PubMed]

7. Liu, G.F.; Li, G.J.; Zhao, H. Efficacy and toxicity of different chemotherapy regimens in the treatment of advanced or metastatic pancreatic cancer: A network Meta-analysis. J. Cell Biochem. 2018, 119, 511-523. [CrossRef]

8. Chin, V.; Nagrial, A.; Sjoquist, K.; O'Connor, C.A.; Chantrill, L.; Biankin, A.V.; Scholten, R.J.; Yip, D. Chemotherapy and radiotherapy for advanced pancreatic cancer. Cochrane Database Syst. Rev. 2018, 3, Cd011044. [CrossRef]

9. Ozaka, M.; Ishii, H.; Sato, T.; Ueno, M.; Ikeda, M.; Uesugi, K.; Sata, N.; Miyashita, K.; Mizuno, N.; Tsuji, K.; et al. A phase Ii study of modified folfirinox for chemotherapy-naïve patients with metastatic pancreatic cancer. Cancer Chemother. Pharmacol. 2018, 81, 1017-1023. [CrossRef]

10. Yoshida, K.; Iwashita, T.; Uemura, S.; Maruta, A.; Okuno, M.; Ando, N.; Iwata, K.; Kawaguchi, J.; Mukai, T.; Shimizu, M. A multicenter prospective phase Ii study of first-line modified folfirinox for unresectable advanced pancreatic cancer. Oncotarget 2017, 8, 111346-111355. [CrossRef]

11. Wang, Z.Q.; Zhang, F.; Deng, T.; Zhang, L.; Feng, F.; Wang, F.H.; Wang, W.; Wang, D.S.; Luo, H.Y.; Xu, R.H.; et al. The efficacy and safety of modified folfirinox as first-line chemotherapy for Chinese patients with metastatic pancreatic cancer. Cancer Commun. 2019, 39, 26. [CrossRef] [PubMed]

12. Mizrahi, J.D.; Rogers, J.E.; Hess, K.R.; Wolff, R.A.; Varadhachary, G.R.; Javle, M.M.; Shroff, R.T.; Ho, L.; Fogelman, D.R.; Raghav, K.P.S.; et al. Modified folfirinox in pancreatic cancer patients age 75 or older. Pancreatology 2020, 20, 501-504. [CrossRef]

13. Junior, U.P.L.S.; Rother, E.T.; Maluf, F.C.; Bugano, D.D.G. Meta-analysis of modified folfirinox regimens for patients with metastatic pancreatic cancer. Clin. Colorectal Cancer 2018, 17, 187-197. [CrossRef]

14. Baldini, C.; Escande, A.; Bouché, O.; el Hajbi, F.; Volet, J.; Bourgeois, V.; Vantroys, T.R.; Ploquin, A.; Desauw, C.; Hebbar, M. Safety and efficacy of folfirinox in elderly patients with metastatic or locally advanced pancreatic adenocarcinoma: A retrospective analysis. Pancreatology 2017, 17, 146-149. [CrossRef] [PubMed]

15. Peter, H.; Macintyre, J.; Kawamura, C.; Maldonado, J.C.; Ernani, V.; Loaiza-Bonilla, A.; Narayanan, G.; Ribeiro, A.; Portelance, L.; Merchan, J.R. A retrospective study of neoadjuvant folfirinox in unresectable or borderline-resectable locally advanced pancreatic adenocarcinoma. BMC Cancer 2012, 12, 1-7.

16. Moorcraft, S.Y.; Khan, K.; Peckitt, C.; Watkins, D.; Rao, S.; Cunningham, D.; Chau, I. FOLFRINOX for locally advanced or metastatic pancreatic ductal adenocarcinoma: The Royal Marsden experience. Clin. Colorectal Cancer 2014, 13, 232-238. [CrossRef] [PubMed]

17. Lyman, G.H.; Yau, L.; Nakov, R.; Krendyukov, A. Overall survival and risk of second malignancies with cancer chemotherapy and G-Csf support. Ann. Oncol. 2018, 29, 1903-1910. [CrossRef]

18. Lyman, G.H.; Reiner, M.; Morrow, P.K.; Crawford, J. The effect of filgrastim or pegfilgrastim on survival outcomes of patients with cancer receiving myelosuppressive chemotherapy. Ann. Oncol. 2015, 26, 1452-1458. [CrossRef]

19. Lyman, G.H.; Dale, D.C.; Culakova, E.; Poniewierski, M.S.; Wolff, D.A.; Kuderer, N.M.; Huang, M.; Crawford, J. The impact of the granulocyte colony-stimulating factor on chemotherapy dose intensity and cancer survival: A systematic review and meta-analysis of randomized controlled trials. Ann. Oncol. 2013, 24, 2475-2484. [CrossRef]

20. Kuderer, N.M.; Dale, D.C.; Crawford, J.; Lyman, G.H. Impact of primary prophylaxis with granulocyte colony-stimulating factor on febrile neutropenia and mortality in adult cancer patients receiving chemotherapy: A systematic review. J. Clin. Oncol. 2007, 25, 3158-3167. [CrossRef]

21. Lalami, Y.; Klastersky, J. Impact of chemotherapy-induced neutropenia (CIN) and febrile neutropenia (FN) on cancer treatment outcomes: An overview about well-established and recently emerging clinical data. Crit. Rev. Oncol. Hematol. 2017, 120, 163-179. [CrossRef]

22. Lee, J.C.; Kim, J.W.; Ahn, S.; Kim, H.W.; Lee, J.; Kim, Y.H.; Paik, K.H.; Kim, J.; Hwang, J.H. Optimal dose reduction of folfirinox for preserving tumour response in advanced pancreatic cancer: Using cumulative relative dose intensity. Eur. J. Cancer 2017, 76, 125-133. [CrossRef] [PubMed] 
23. Sohal, D.P.S.; Kennedy, E.B.; Khorana, A.; Copur, M.S.; Crane, C.H.; Garrido-Laguna, I.; Krishnamurthi, S.; Moravek, C.; O’Reilly, E.M.; Philip, P.A.; et al. Metastatic pancreatic cancer: Asco clinical practice guideline update. J. Clin. Oncol. 2018, 36, 2545-2556. [CrossRef] [PubMed]

24. Okusaka, T.; Ikeda, M.; Fukutomi, A.; Ioka, T.; Furuse, J.; Ohkawa, S.; Isayama, H.; Boku, N. Phase Ii study of folfirinox for chemotherapy-naïve Japanese patients with metastatic pancreatic cancer. Cancer Sci. 2014, 105, 1321-1326. [CrossRef]

25. Muranaka, T.; Kuwatani, M.; Komatsu, Y.; Sawada, K.; Nakatsumi, H.; Kawamoto, Y.; Yuki, S.; Kubota, Y.; Kubo, K.; Kawahata, S.; et al. Comparison of efficacy and toxicity of folfirinox and gemcitabine with Nab-paclitaxel in unresectable pancreatic cancer. J. Gastrointest. Oncol. 2017, 8, 566-571. [CrossRef] [PubMed]

Publisher's Note: MDPI stays neutral with regard to jurisdictional claims in published maps and institutional affiliations.

(C) 2020 by the authors. Licensee MDPI, Basel, Switzerland. This article is an open access article distributed under the terms and conditions of the Creative Commons Attribution (CC BY) license (http://creativecommons.org/licenses/by/4.0/). 